\title{
Highly Soluble TRPV4 Antagonists for Oral \& IV Delivery
}

\author{
Brian Lawhorn \\ March 25, 2020
}

All studies were conducted in accordance With the GSK policy on the Care, Welfare And Treatment of Laboratory Animals and were reviewed by the Institutional Animal Care and Use Committee either At GSK or by the ethical review process at the Institution where the work was performed. 


\section{TRPV4}

Transient Receptor Potential Vanilloid-4 Sensory Protein

- Non-selective $\left(\mathrm{Na}^{+}, \mathrm{Ca}^{2+}\right)$ ion channel

- Responsive to pressure, heat, osmolarity $\mathrm{pH}$ and endogenous ligands

- Regulates alveolar-septal barrier permeability

- Potential for CHF \& respiratory indications
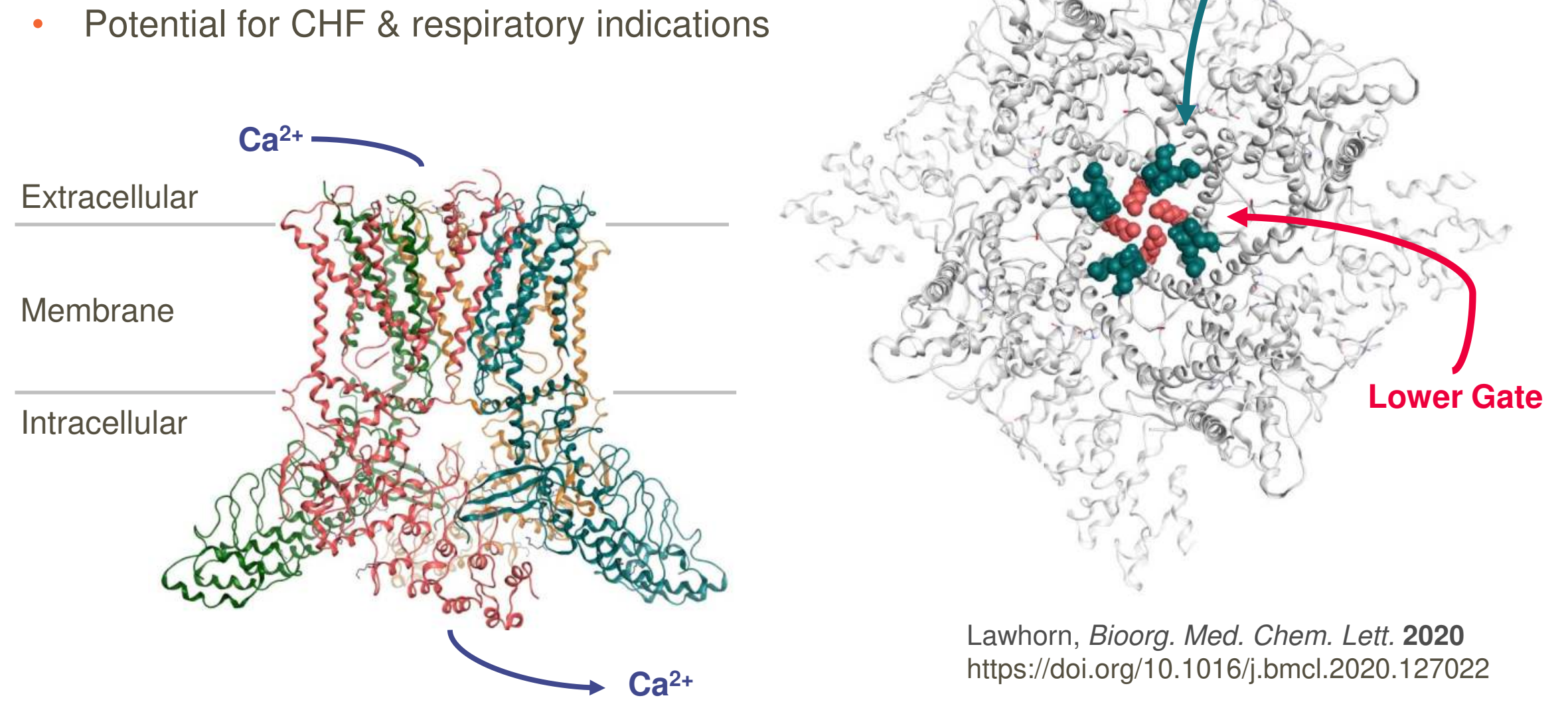

Lawhorn, Bioorg. Med. Chem. Lett. 2020 https://doi.org/10.1016/j.bmcl.2020.127022

Hite \& Yuan, Nature Struct. Mol. Biol. 2018, 25, 252; Van Horn, Acc. Chem. Res. 2019, 52, 1643 


\section{TRPV4 and Heart Failure}

Effects of TRPV4 Activation in Pulmonary Vasculature

\section{Congestive Heart Failure}

- \#1 cause of hospitalization in elderly

- Disrupted lung / vasculature barrier

- Pulmonary congestion

- Shortness of breath

\section{TRPV4}

- Responsive to vasculature pressure

- Regulates endothelial cell permeability

- Agonists cause lung edema \& death

- Antagonists prevent HF-induced lung edema in animal models
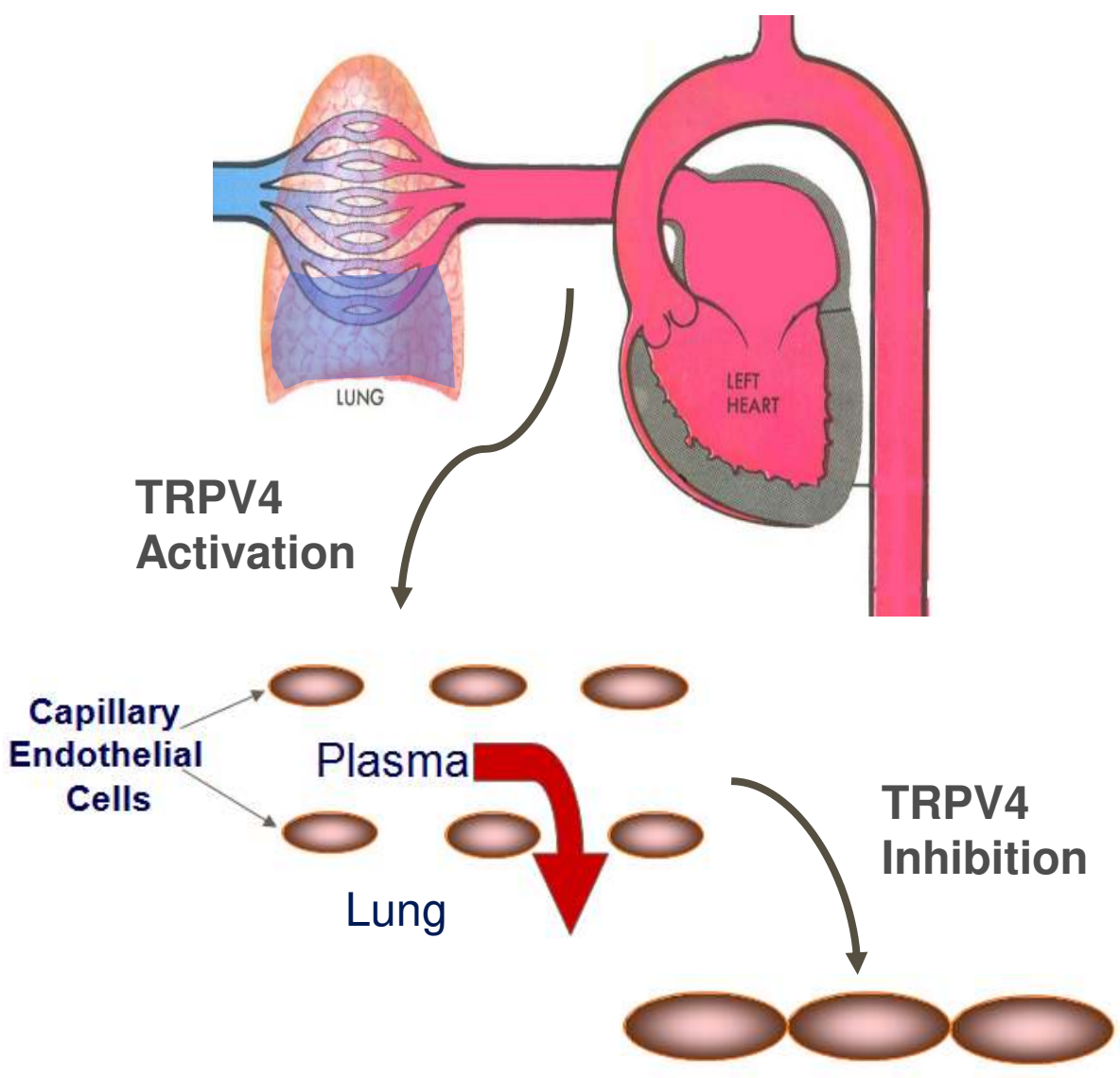

Plasma

Lung 


\section{GSK TRPV4 Program}

Seeking Backup Candidate from a Novel Chemotype

\section{GSK2798745}

- First-in-class TRPV4 Ion Channel Inhibitor

- Entered Phase 2 in 2018

- Heart Failure \& Respiratory indications

Brooks, ACS Med Chem Lett. 2019, 10, 1228

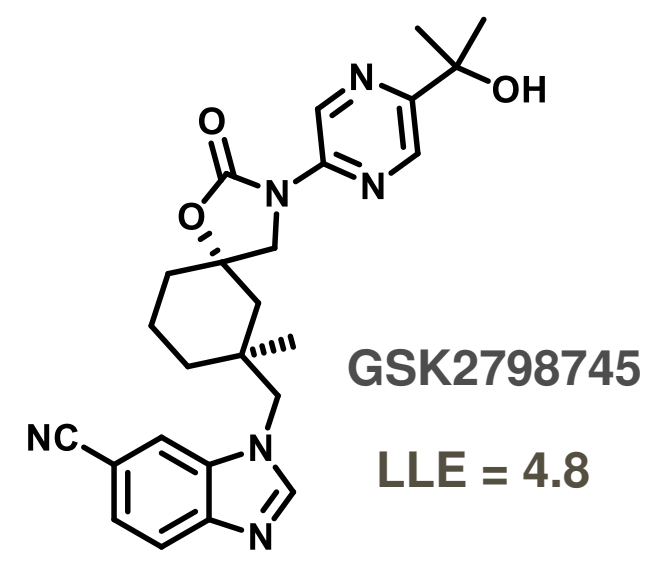

\section{$2^{\text {nd }}$ Generation Drug Candidate}

- Novel chemotype

- Drug-like properties

- Specificity for TRPV4

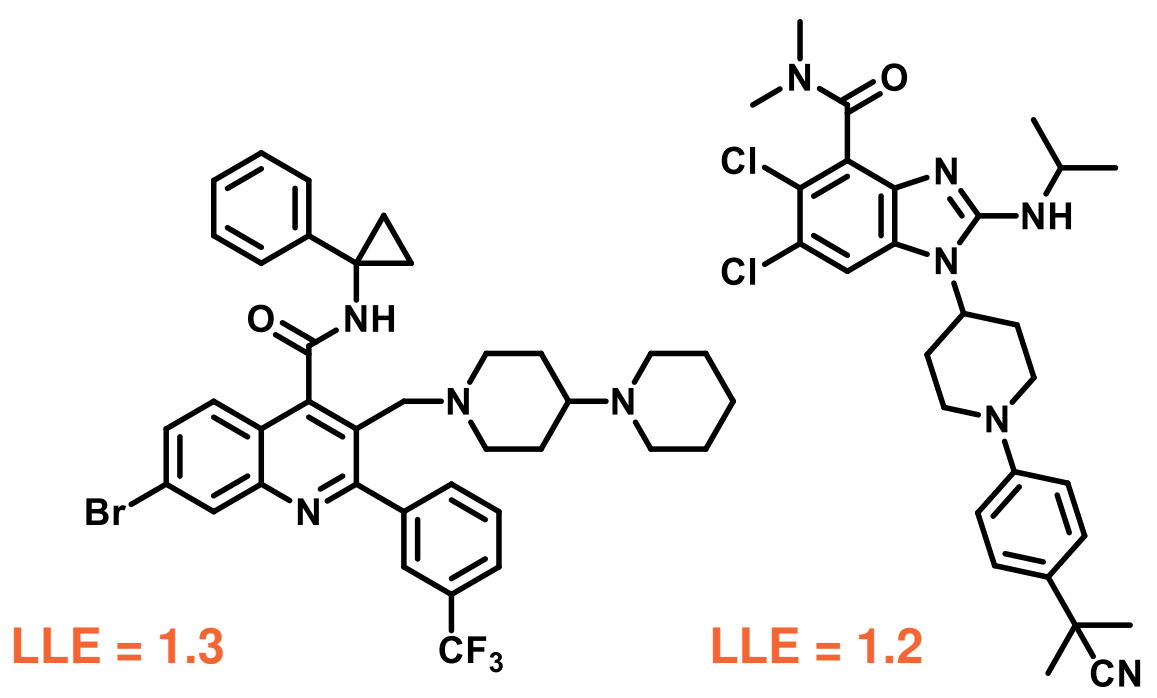




\section{Sulfonamide TRPV4 Antagonists}

\section{Poor Solubility - An Unintended Consequence of LLE Optimization}

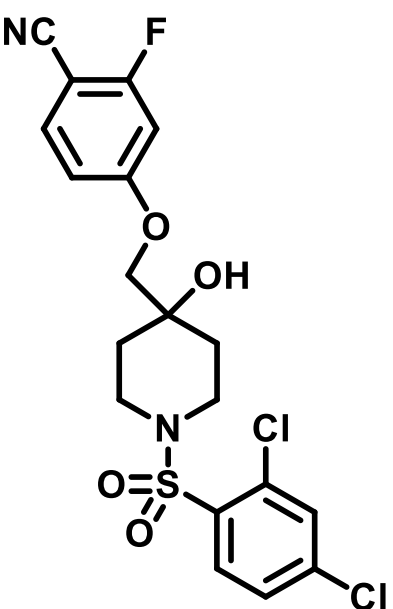

$\mathrm{IC}_{50}=126 \mathrm{nM}$

ChromLogD $=6.3$

LLE $=0.6$

FaSSIF $=17 \mu \mathrm{g} / \mathrm{mL}$
Conformational Restriction

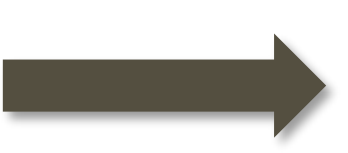

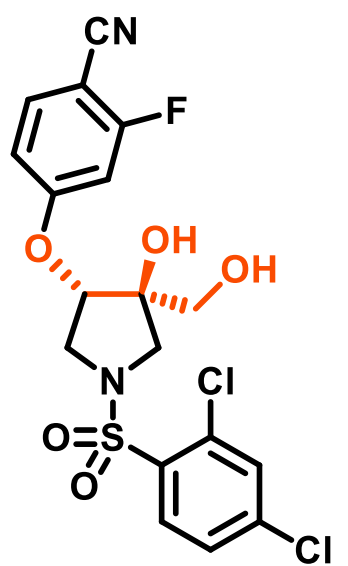

$\mathrm{IC}_{50}=3 \mathrm{nM}$

ChromLogD $=4.8$

LLE $=3.7$

FaSSIF $=19 \mu \mathrm{g} / \mathrm{mL}$
Pharmacokinetic Optimization

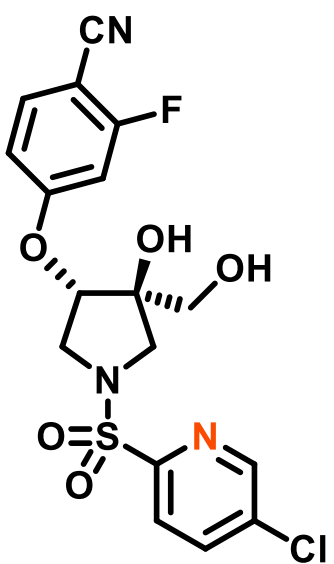

$\mathrm{IC}_{50}=34 \mathrm{nM}$

ChromLogD = 3.4

LLE = 4.1

FaSSIF $=26 \mu \mathrm{g} / \mathrm{mL}$

- Goal of LLE (LipE) optimization: identify compounds with drug-like properties

- Optimization of LLE via entropic effects can produce agents with poor solubility 


\section{Origin of Poor Solubility}

\section{Excessive stability of crystalline material}

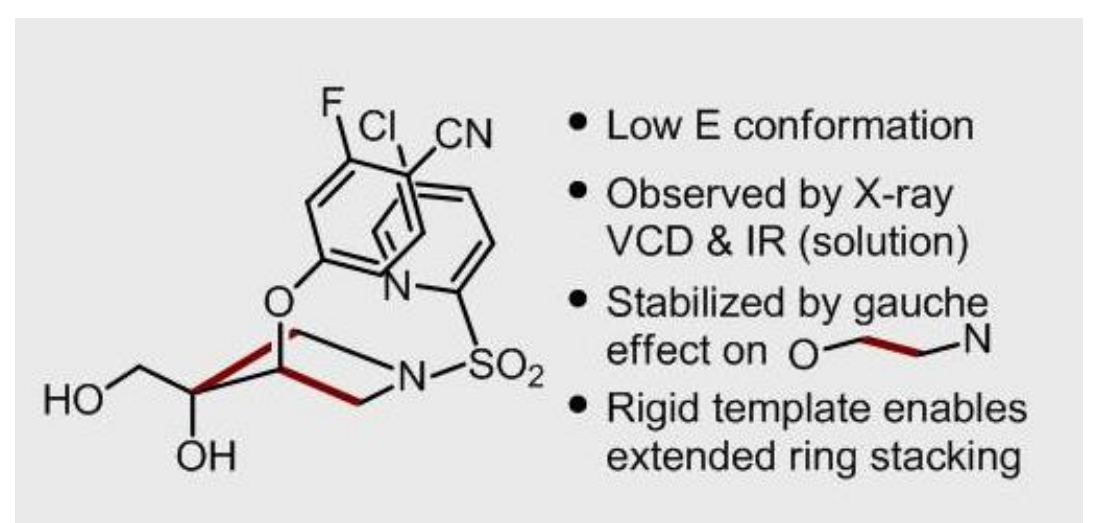

\section{Poor solubility resulting from}

- Reduced entropy of solvation

- High crystal lattice energy

- Extended ring stacking within crystal

- Stabilizing hydrogen bond network

\section{Small Molecule X-ray Crystal Structure}
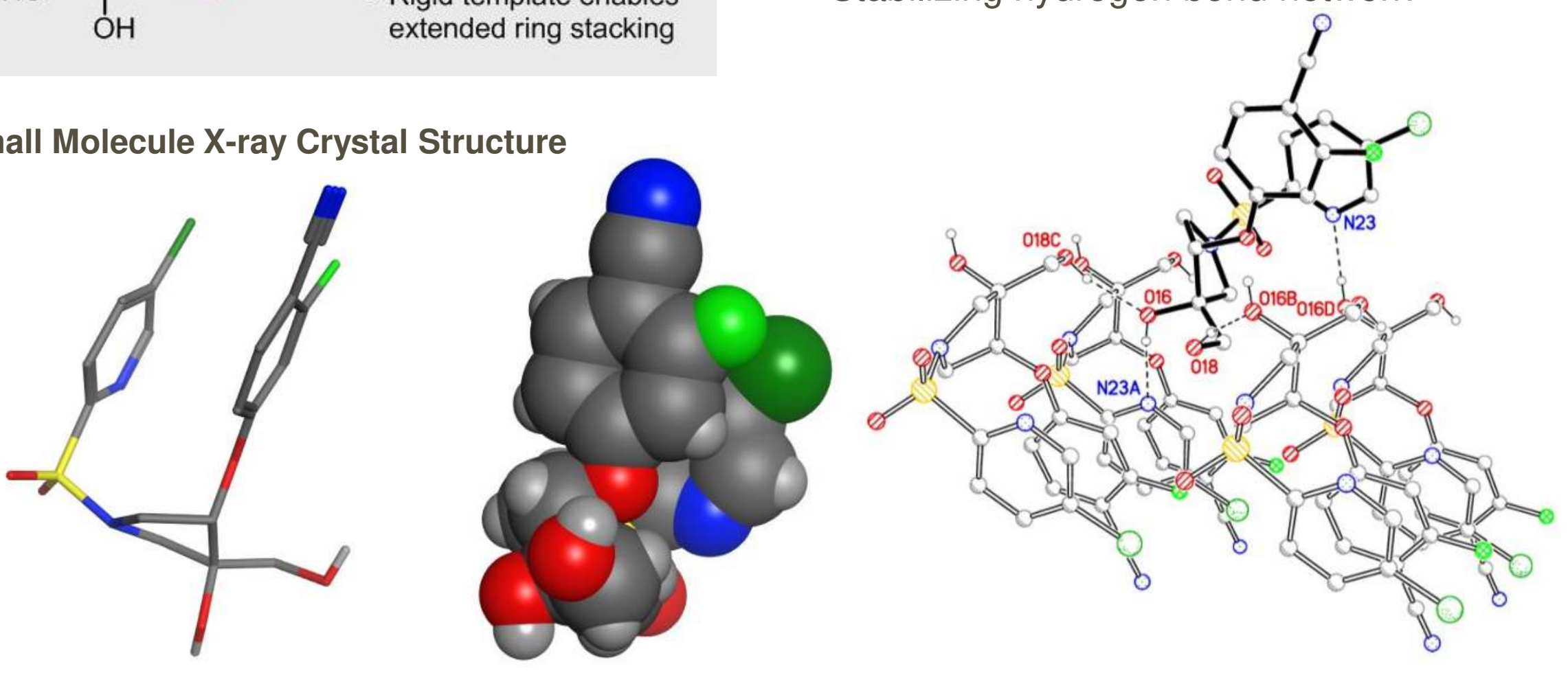


\section{Oral Bioavailability of Suspension Doses}

Poor solubility limits dose escalation required for safety studies

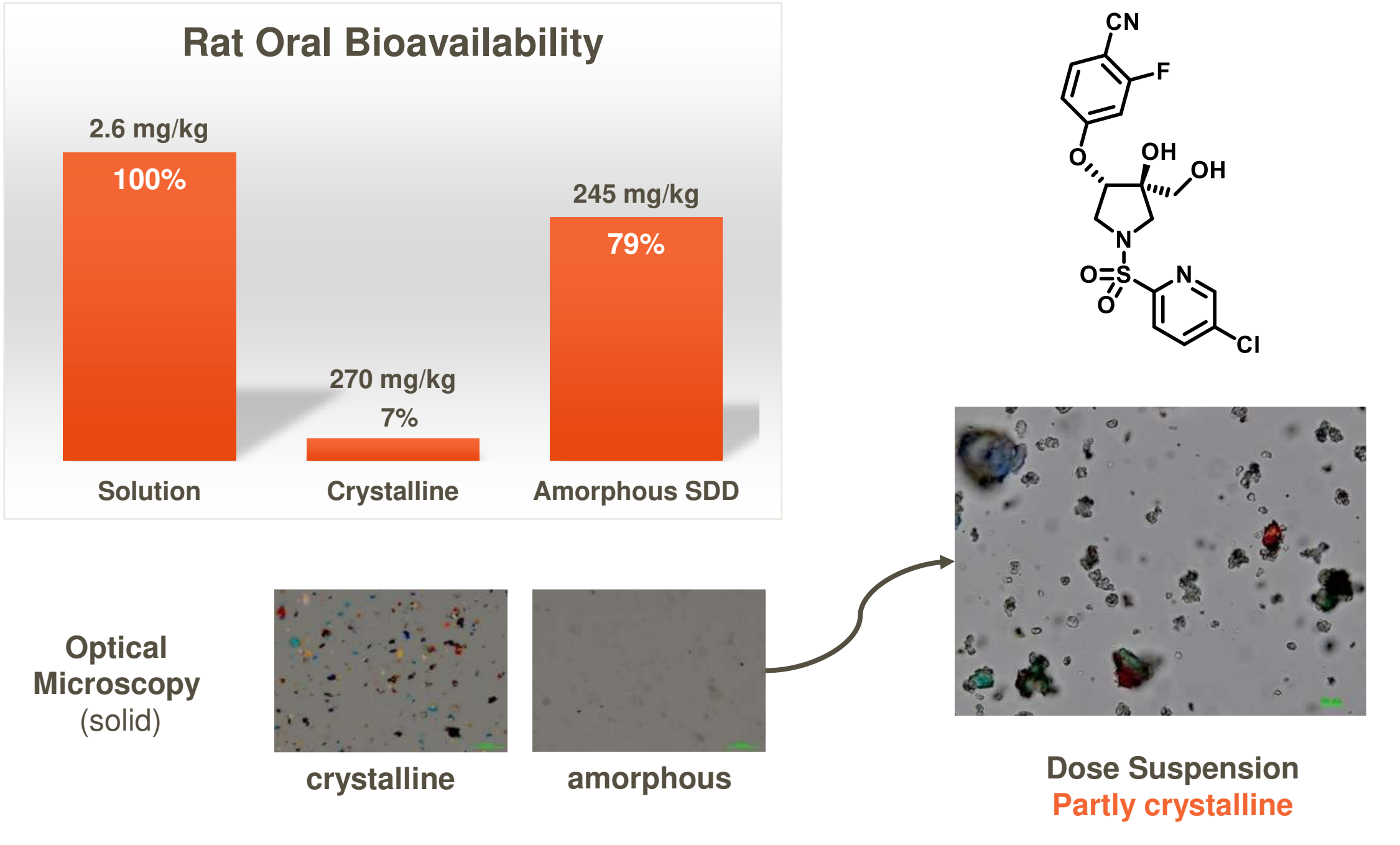




\section{Strategies to Improve Solubility}

\section{Reducing Conformational Bias}

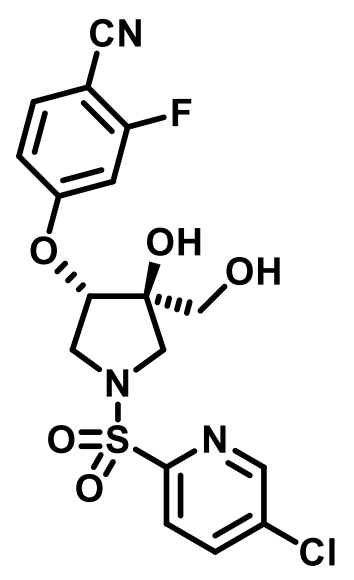

$\mathrm{IC}_{50}=34 \mathrm{nM}$

ChromLog $\mathrm{D}=3.4$

FaSSIF $=26 \mu \mathrm{g} / \mathrm{mL}$
Conformational

Relaxation

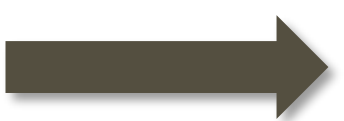

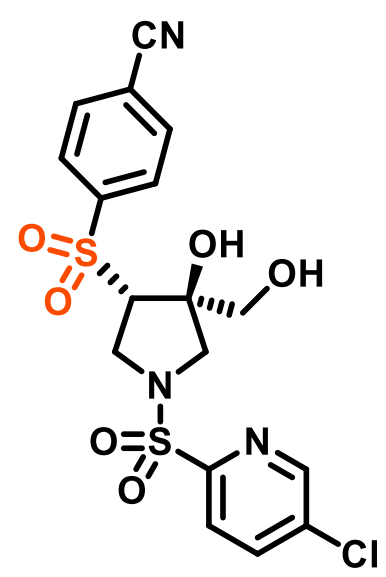

$\mathrm{IC}_{50}=3800 \mathrm{nM}$

ChromLog $\mathrm{D}=3.1$

FaSSIF $>1000 \mu \mathrm{g} / \mathrm{mL}$

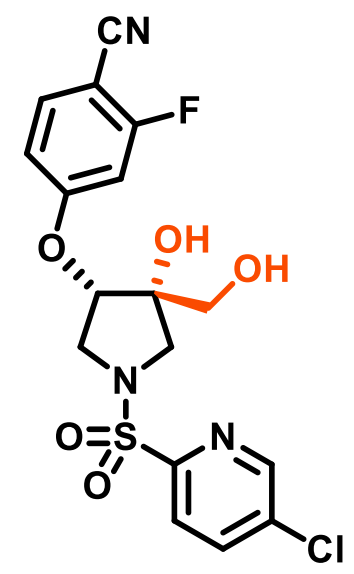

$\mathrm{IC}_{50}=190 \mathrm{nM}$

ChromLog $\mathrm{D}=3.5$ FaSSIF $=130 \mu \mathrm{g} / \mathrm{mL}$
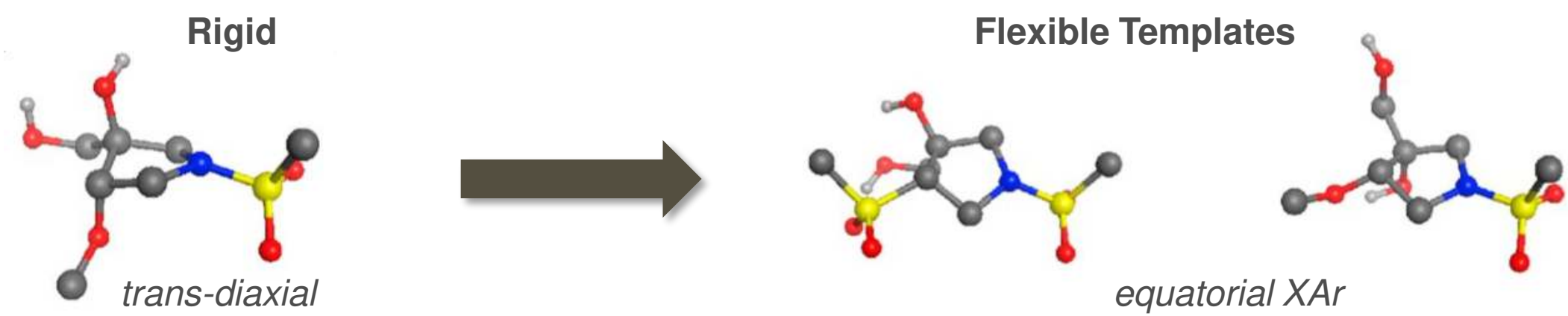

- Crystalline stability arises from strong conformational bias of trans-pyrrolidine diol 


\section{Strategies to Improve Solubility}

Solubilizing Prodrug

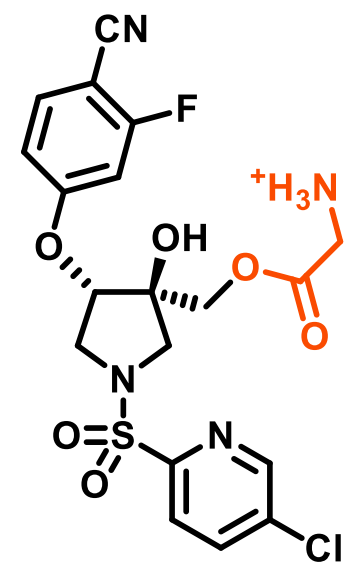

Prodrug

ChromLog $\mathrm{D}=3.1$

FaSSIF $=542 \mu \mathrm{g} / \mathrm{mL}$
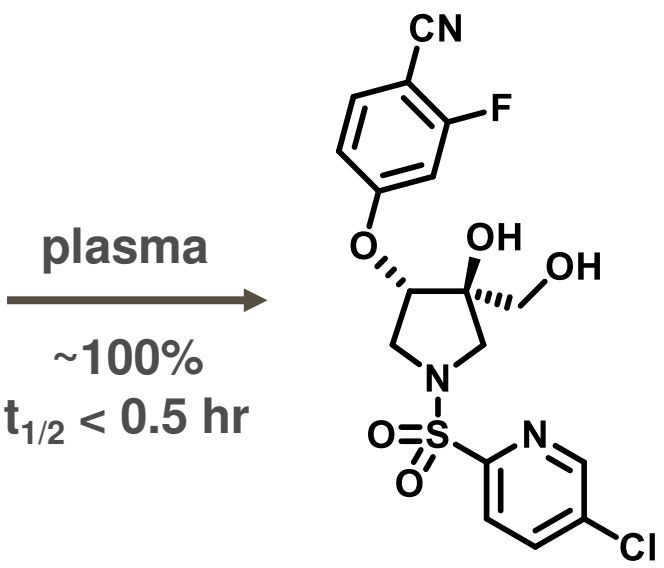

$\mathrm{IC}_{50}=34 \mathrm{nM}$

ChromLogD = 3.4

FaSSIF $=26 \mu \mathrm{g} / \mathrm{mL}$

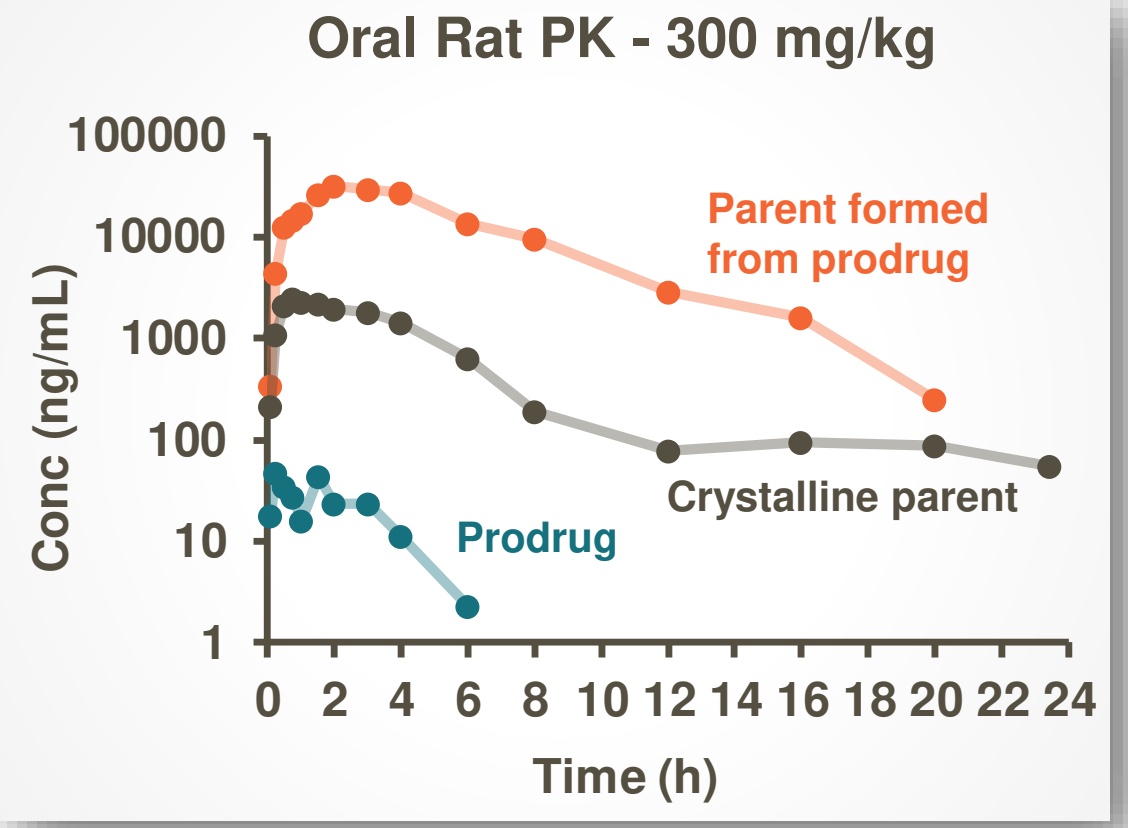

Oral Rat PK - 300 mg/kg

\section{7d Rat Safety Findings for Prodrug}

- Kidney stones \& dilated tubules

- Liver hypertrophy, $\uparrow$ billirubin, $\uparrow A L P$

Obstructive liver \& kidney effects may be caused by crystallization of parent in vivo 


\section{Strategies to Improve Solubility}

Charged, Stable Analogues

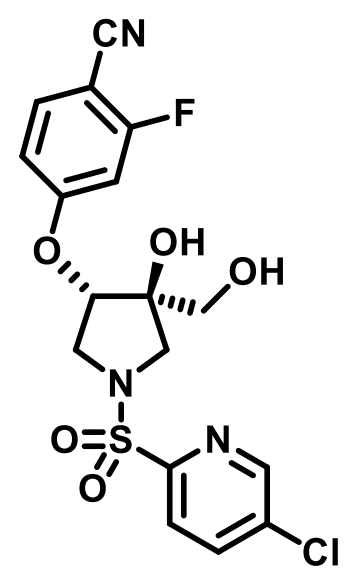

$\mathrm{IC}_{50}=34 \mathrm{nM}$

ChromLog $\mathrm{D}=3.4$

FaSSIF $=26 \mu \mathrm{g} / \mathrm{mL}$
Introduce Stable

Charged Group

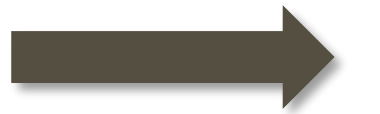

Safety Risks

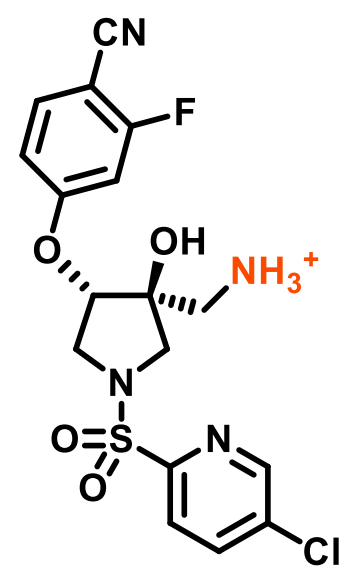

$\mathrm{IC}_{50}=12 \mathrm{nM}$

ChromLog $\mathrm{D}=2.5$

FaSSIF $>1000 \mu \mathrm{g} / \mathrm{mL}$

hERG $=20 \mu \mathrm{M}$

Cyp3A4 $=12 \mu \mathrm{M}$

CAD-likeness $=51$

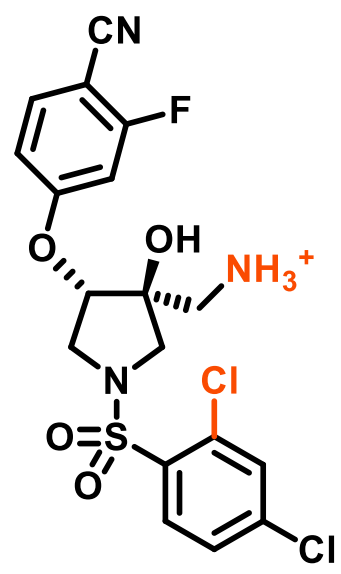

$\mathrm{IC}_{50}=3.5 \mathrm{nM}$

ChromLog $\mathrm{D}=4.0$

FaSSIF $=78 \mu \mathrm{g} / \mathrm{mL}$

hERG $=3 \mu \mathrm{M}$

Cyp3A4 $=1.6 \mu \mathrm{M}$

CAD-likeness $=61$

- Solubility of amino alcohol template is governed by lipophilicity, unlike the trans-diol core

- Controlling lipophilicity is critical to attenuate off-target activities of the basic amines 


\section{GSK3527497 - A Highly Soluble TRPV4 Candidate}

Novel chemotype - suitable for oral and iv delivery

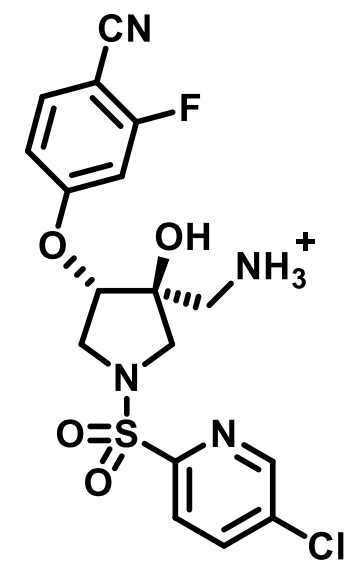

GSK3527497
Pharmacokinetics

\begin{tabular}{|c|c|c|}
\hline Species & Rat & Dog \\
\hline CL $(\mathrm{ml} / \mathrm{min} / \mathrm{kg})$ & 45 & 5.9 \\
\hline Vdss $(\mathrm{L} / \mathrm{kg})$ & 6.7 & 5.9 \\
\hline MRT $(\mathrm{hr})$ & 2.5 & 17 \\
\hline Oral F $(\%)$ & 26 & 84 \\
\hline PPB (\% free) & 14 & 32 \\
\hline Proj. Human Dose & $150 \mathrm{mg}$ bid & $10 \mathrm{mg} \mathrm{qd}$ \\
\hline
\end{tabular}

Pharmacology \& Safety

- Prevents lung edema due to TRPV4 activation

- Ames-negative

- $84 \%$ Oral F @ 100 mg/kg

- Suitable safety profile in rodents \& non-rodents

- Novel drug candidate

\section{Key Lessons}

- Entropically-driven LLE optimization identifies selective leads, with poor solubility

- In extreme cases, poorly soluble compounds may crystallize in vivo causing toxicity

- Entropically-optimized templates with solubilizing amino groups have drug-like properties 


\section{Synthesis of GSK3527497}
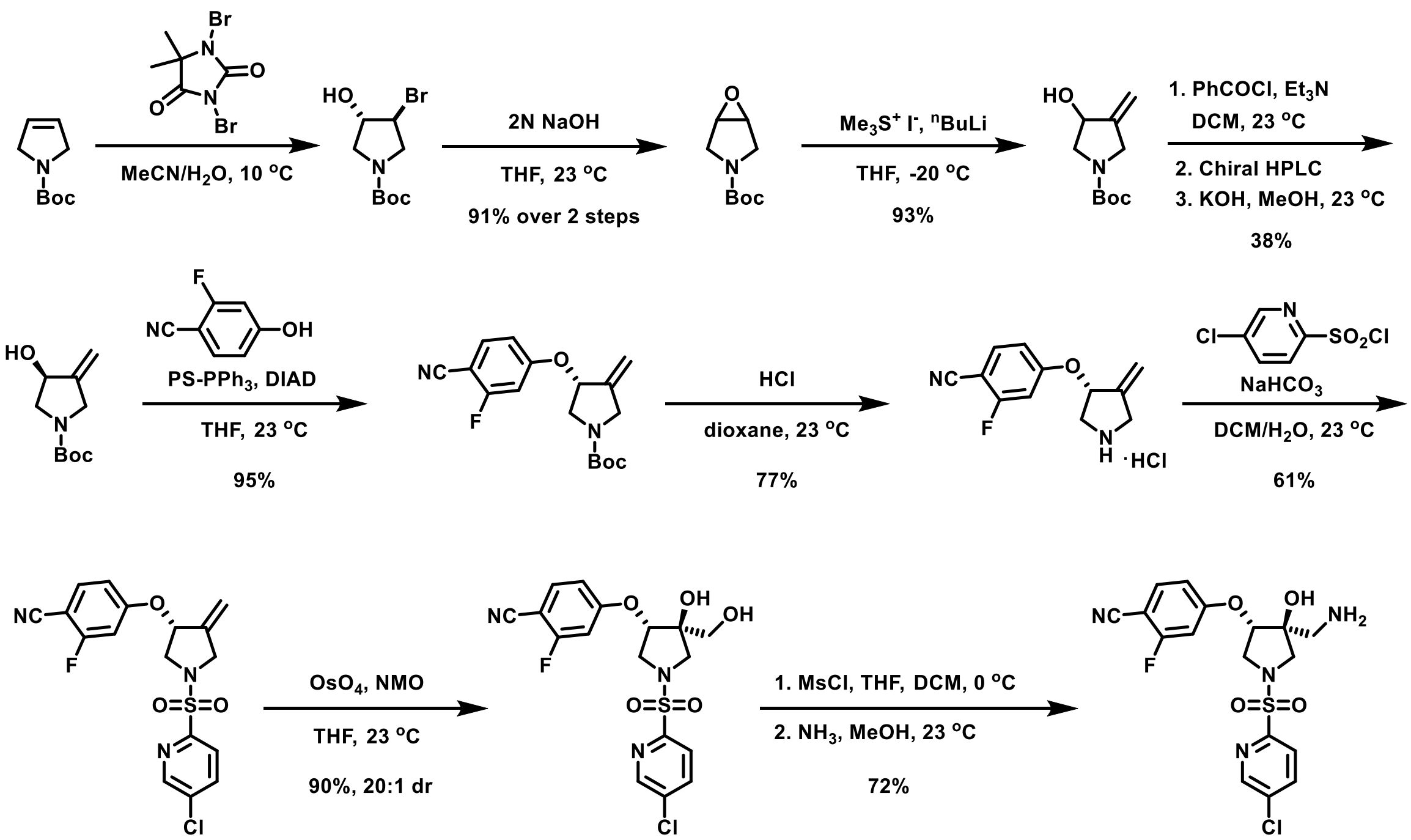


\section{Medicinal Chemistry}

- Linda Barton

- Eric Boros

- Carl Brooks

- Edward Brnardic

- Brian Budzik

- David Cowan

- Dennis Holt

- Huijie Li

- Peng Li

- Jay Matthews

- Jeff McAtee

- Brent McCleland

- Chris Neipp

- Jaclyn Patterson

- Israil Pendrak
Medicinal Chemistry

- Joe Pero

- Ralph Rivero

- Bob Sanchez

- Matt Sender

- Raynold Shenje

- Art Shu

- Patrick Stoy

- Lamont Terrell

- Guosen Ye

- Mark Youngman

Biology \& Safety

- Andy King

- Dave Behm

- Xiaoping Xu

- Melissa Costell

\section{DMPK}

- Kevin Baptiste

- Stephen Eisennagel

- Larry Jolivette

- Barbara Priebe

- Theresa Roethke

Screening \& Technology

- Roy Copley

- Karl Erhard

- Mike Fischer

- Amy Gibble

- Mike Klein

- Ashley Leister

- Grazyna Graczyk-Millbrandt

- Doug Minnick

- James Thomas 